\title{
Electric-field-dependent variable-range hopping conduction in $\mathrm{PrBa}_{2} \mathrm{Cu}_{3} \mathrm{O}_{7-y}$
}

\section{R. B. Thompson \& M. Singh}

To cite this article: R. B. Thompson \& M. Singh (1997) Electric-field-dependent variablerange hopping conduction in $\mathrm{PrBa}_{2} \mathrm{Cu}_{3} \mathrm{O}_{7-y}$, Philosophical Magazine B, 75:2, 293-302, DOI: 10.1080/13642819708202317

To link to this article: https://doi.org/10.1080/13642819708202317

曲 Published online: 20 Aug 2006.

Submit your article to this journal ¿

山 Article views: 50

Q View related articles $₫$

This is an Accepted Manuscript of an article published by Taylor \& Francis in Philosophical Magazine B on 1997-02-01, available online: http://www.tandfonline.com/10.1080/13642819708202317. 


\title{
Electric-field-dependent variable-range hopping conduction in $\mathrm{PrBa}_{2} \mathrm{Cu}_{3} \mathrm{O}_{7-y}$
}

\author{
By R. B. Thompson and M. Singh \\ Department of Physics, University of Western Ontario, London, \\ Canada N6A 3K 7
}

[Received 20 June 1996 and accepted 31 July 1996]

\begin{abstract}
The variable-range hopping conductivities of quasi-two-dimensional systems in electric fields were calculated for the cases of both constant and energydependent densities of states. The localized states were considered to be randomly distributed in space and energy coordinates, and the carriers were considered to be distributed according to the Fermi distribution. Approximations yielded analytic results valid for most temperatures and electric fields and included localized states both above and below the Fermi level. We hypothesized that the localized states of $\mathrm{PrBa}_{2} \mathrm{Cu}_{3} \mathrm{O}_{7-y}$ (PBCO) are distributed in the CuO planes, making that compound behave as a quasi-twodimensional material. We compared our theory with experiments of PBCO-based junctions and found good agreement between theory and experiment.
\end{abstract}

\section{§1. INTRODUCTION}

Superconducting junctions (such as superconductor-normal metal (S-N) or superconductor-normal metal-superconductor ( $\mathrm{S}-\mathrm{N}-\mathrm{S}$ junctions) have many current and potential future applications. Among these are superconducting quantum interference devices and superconducting computer components. It is therefore important that their properties are fully understood. While this is already the case for conventional low-temperature superconductors, the high-temperature superconductor (HTS) oxide junctions show some peculiar behaviour. Since the HTS materials have obvious advantages and are being incorporated into modern technology, it is imperative that any unexpected behaviour be accounted for. A material used recently as the $\mathrm{N}$ layer between the high-temperature superconductors is $\mathrm{PrBa}_{2} \mathrm{Cu}_{3} \mathrm{O}_{7-y}$ (PBCO). It is well latticed matched to the popular $\mathrm{YBa}_{2} \mathrm{Cu}_{3} \mathrm{O}_{7-y}$ (YBCO) superconductor and remains non-superconducting unlike other substitutions for Y. PBCO has therefore been the subject of some recent experimental and theoretical work (Kabasawa et al. 1993, Singh, Tarutani, Kabasawa and Takagi 1994a,b, Singh, Thompson and Dumas 1996, van Ancum, Verhoeven, Blank and Rogalla 1995), both because of its utility in HTS junctions, and because the study of PBCO properties may illuminate the causes of the depression of superconductivity in Y'BCO on substituting Pr for Y.

There has been considerable interest in the study of the electric field and temperature dependent conductivity of PBCO-based S-N-S junctions. These experiments show a variable-range hopping (VRH) type of conduction (Seong, Lee, and Youm 1990, Kabasawa et al. 1992, 1993, Boguslavskij et al. 1994, Koyanagi et al. 1994). Kabasawa et al. (1993) have fabricated $\mathrm{S}-\mathrm{N}-\mathrm{S}$ junctions where $\mathrm{S}$ is 
$\mathrm{HoBa}_{2} \mathrm{Cu}_{3} \mathrm{O}_{7-y}$ and $\mathrm{N}$ is $\mathrm{PBCO}$. They have measured the temperature and electricfield-dependent conductivity of the PBCO junctions and films and have observed a VRH type of conduction. Kabasawa et al. (1993) and Singh et al. (1994a,b) used superconducting electrodes in their experiments. More recentiy, van Ancum et al. (1995) studied PBCO thin films using noble-metal electrodes and the transmission line model to remove the effects of superconducting electrodes. According to them, superconducting electrodes can cause superconducting coupling or pair breaking below the critical temperature $T_{\mathrm{c}}$. Their method also allows measurements above the $T_{\mathrm{c}}$ of the electrodes. They also observed a VRH mechanism of electrical conduction.

Apsley and Hughes $(1974,1975 \dagger)$ published a theory for VRH in bulk (threedimensional) materials, as did Pollak and Riess (1976) and Shklovskii (1976). Singh et al. (1994a,b) used the Apsley-Hughes method to develop a theory for VRH in quasi-two-dimensional systems and hypothesized that the localized states involved in conduction are located in the quasi-two-dimensional $\mathrm{CuO}$ planes of PBCO. The theory of Singh et al. is able to explain the temperature-dependent conductivity of PBCO thin film, but it is not able to explain the electric-field-dependent conductivity experiments (Kabasawa et al. 1992, 1993) for $\beta>1$; the quantity $\beta$ is proportional to the electric field $E$ and inversely proportional to the temperature $T$. It is defined as $\beta \equiv q E / 2 \alpha k_{\mathrm{B}} T$, where $\alpha$ is the localization length, $q$ is the charge and $k_{\mathrm{B}}$ is Boltzmann's constant. Recently, we have studied the electric-field-dependent VRH conductivities of quasi-two-dimensional systems with a constant density of states (DOS) (Singh et al. 1996). We calculated the expression of the nearest-neighbour hopping distance $R_{\mathrm{nn}}$ as a function of electric field and temperature. We then put the expression for $R_{\mathrm{nn}}$ into the Mott expression (Mott and Davis 1971, Mott 1974) $\sigma=\sigma_{0} \exp \left(-R_{\mathrm{nn}}\right)$ to calculate the conductivity and compared our theory with the experiments of Kabasawa et al. (1993). The theoretical results are valid for a wide range of $\beta$, including $\beta>1$. We neglected the effect of electron-electron interactions in the conductivity calculation. It is known from electronic and optical properties of $\mathrm{YBCO}$ and $\mathrm{PBCO}$ compounds that the electron-electron interaction plays an important role in understanding the above properties (Anderson 1988, Sinha and Singh 1988, Singh and Sinha 1989, Forro 1994).

The aim of the present paper is to study the role of electron-electron interaction in the electric-field-dependent VRH mechanism of PBCO-based $\mathrm{S}-\mathrm{N}-\mathrm{S}$ junctions and thin films. We shall include the effect of the electron-electron interaction through the DOS of localized states in $\mathrm{CuO}$ planes of $\mathrm{PBCO}$. Instead of using the simplified Mott expression of conductivity, we shall use the method developed by Singh et al. (1994a,b) to calculate the conductivity. We consider that the localized states are randomly distributed in both space and energy coordinates, and we let the charge carriers hop between these states in both coordinates. We let the carriers be distributed according to the Fermi distribution function and consider localized states both above and below the Fermi level. This allowed us to derive an expression for the number of vacant states within a certain range of the carrier, and in turn that number allowed us to derive an energy-dependent mobility. The mobility was then integrated over energy to yield conductivity expressions. From all this we got new expressions that improve upon the constant-DOS approximation previously

tWe found that the denominator of eqn. (15) of this reference is incorrect. It should be $1 / 3(1+\beta)+1 / 3+\beta / 3$. 
obtained by Singh et al. (1996) based on Mott's method. Our expressions are valid for most $\beta$ values and include pre-exponential factors ignored in previous conductivity expressions (Singh et al. 1996). We have compared our theory with the experimental PBCO-based S-N-S junction work of Kabasawa et al. (1992, 1993). Good agreement between theory and experiment was found. Since our model is generally valid for any two-dimensional VRH system, we further tested it by comparing it with the data of a quasi-two-dimensional sample of $\mathrm{Bi}_{14} \mathrm{Te}_{11} \mathrm{~S}_{10}$ (Liu and Soonpaa 1993).

\section{§ 2. VARIABLE-RANGE HOPPING CONDUCTIVITY}

To evaluate the VRH conductivity for bulk (three-dimensional) materials, Mott and Davis (1971), Mott (1974), and Apsley and Hughes (1974, 1975) assumed that the DOS of localized electrons is constant and does not depend on energy. Later Shklovskii $(1973,1991)$ and Efros and Shklovskii (1985) showed that, if one includes the effect of electron-electron interactions in VRH conductivity, the DOS is not constant but depends on the energies of the localized electrons. They found that

$$
D(\omega)= \begin{cases}D_{0}, & \omega>\frac{\Delta_{C g}}{k_{\mathrm{B}} T}, \\ D_{1} k_{\mathrm{B}} T \omega^{d-1}, & \omega<\frac{\Delta_{C g}}{k_{\mathrm{B}} T},\end{cases}
$$

where $\Delta_{C g}$ is called the Coulomb gap, $d$ is the dimensionality of the system, and $D_{0}$ and $D_{1}$ are constants. Here energy is measured with respect to the Fermi energy. Note that, at the Fermi level, the above DOS reduces to zero, which contradicts the approximation made by Mott. There is experimental evidence for the existence of the Coulomb gap in two dimensional systems (Hu et al. 1995, Mason, Kravchenko, Bowker and Furneaux 1995).

Here we considered that, in the $\mathrm{CuO}$ plane of $\mathrm{PBCO}$, localized states are randomly distributed in energy and space coordinates (hopping space) and form a discrete array of sites. The probability of a charge carrier hopping from an initial state to a final state in the hopping space is given by (Apsley and Hughes 1974, 1975, Mott 1974, Mott and Davis 1971, Singh et al. 1994 a,b)

$$
W(R)=W_{0} \exp (-R)
$$

where $W_{0}$ is a constant and $R$ is the distance between two states in the energy-space coordinates and is called the range. In the presence of an electric field, the range is given as (Singh et al. 1994a,b)

$$
R= \begin{cases}x(1+\beta \cos \theta)+\omega-\varepsilon, & \varepsilon<\omega+x \beta \cos \theta, \\ x, & \varepsilon>\omega+x \beta \cos \theta,\end{cases}
$$

where $\beta=q E / 2 \alpha k_{\mathrm{B}} T$. Here $q$ is the charge of an electron and $x$ is a distance between two sites in the space coordinates. $\varepsilon$ and $\omega$ are the energy variables of the initial and the final sites respectively. $\theta$ is an angle between the space variable $x$ and the electric field $E$. Note that the space and energy variables are presented in reduced coordinates (Singh et al. 1994a,b). The reduced coordinate $x$ should be multiplied by $1 / 2 \alpha$ to express it in distance units. Similarly, $\varepsilon$ and $\omega$ should be multiplied by $k_{\mathrm{B}} T$ to write them in energy units. $\alpha$ is the inverse of the localization length.

In the presence of an electric field, the spatial displacement of electrons in the hopping space is more likely to be downfield than upfield. Let $x_{\mathrm{f}}$ be the average spatial distance travelled in the direction of the electric field and $W\left(R_{n n}\right)$ be the 
hopping probability for the average nearest-neighbour hopping distance $R_{\mathrm{nn}}$. Then $\nu_{\mathrm{p}} x_{\mathrm{f}} W\left(R_{\mathrm{nn}}\right) / 2 \alpha$ is the average spatial distance travelled per unit time by a carrier in the direction of the electric field. The mobility is obtained by dividing this quantity by the electric field (Singh et al. 1994a,b) and is thus

$$
\mu=\frac{\nu_{\mathrm{p}}}{2 \alpha E} x_{\mathrm{f}} \exp \left(-R_{\mathrm{nn}}\right)
$$

where $\nu_{\mathrm{p}}$ is the hopping attack frequency which may be taken as a phonon frequency and $x_{\mathrm{f}}$, which is expressed in reduced units, is given in the articles by Singh et al. $(1994 a, b)$ as

$$
x_{\mathrm{f}}=\left(\int \cos \theta \mathrm{d} \theta \int_{-\infty}^{R_{\mathrm{nn}}+\varepsilon} \mathrm{d} \omega D(\omega)[1-f(\omega)] x^{2}\right) /\left(\int \mathrm{d} \theta \int_{-\infty}^{R_{\mathrm{nn}}+\varepsilon} \mathrm{d} \omega D(\omega)[1-f(\omega)] x\right) .
$$

$D(\omega)$ is the DOS of localized carriers in two-dimensional systems and $f(\omega)$ is the Fermi distribution function. The expression for the nearest-neighbour hopping distance $R_{\mathrm{nn}}$ is written as (Apsley and Hughes 1975, Singh et al. 1994a,b)

$$
R_{\mathrm{nn}}=\int_{0}^{\infty} R P_{\mathrm{nn}}(R) \mathrm{d} R
$$

where $P_{\mathrm{nn}}(R)$ is the probability distribution of the nearest neighbours in the hopping space which is defined as

$$
P_{\mathrm{nn}}(R)=\Delta N(R) \exp [-N(R)]
$$

$N(R)$ is the number of vacant sites within the range $R$ for the nearest-neighbour sites and $\Delta N(R) \equiv \partial N / \partial R$ is the number of localized states between $R$ and $R+\mathrm{d} R$.

For quasi-two-dimensional systems, the number $N(R)$ of vacant sites within a range $R$ as a function of temperature and electric field is given by (Singh et al. $1994 a, b)$.

$$
N(R)=\frac{k_{\mathrm{B}} T}{4 \alpha^{2}} \int_{0}^{2 \pi} \mathrm{d} \theta \int_{0}^{R} x \mathrm{~d} x \int_{-\infty}^{\omega_{\max }} D(\omega)[1-f(\omega)] \mathrm{d} \omega,
$$

where $x_{\max }=R$ and $\omega_{\max }=R+\varepsilon-x(1+\beta \cos \theta)$. These are obtained from eq. (3). Note that $N$ is a function of electric field $E$, temperature $T$ and initial energy $\varepsilon$. The expression of $N$ for a constant DOS has been evaluated in our previous paper (Singh et al. 1996) as

$$
N_{\mathrm{c}}=\frac{T}{T_{\mathrm{c}}^{\prime}} R^{3}\left[\left(\eta_{1}+\frac{1}{2}+\frac{2 \beta}{\pi}\right)+\left(\frac{3}{2}+3 \eta_{1}\right) \frac{\varepsilon}{R}\right]
$$

where the subscript c denotes a constant DOS. Similarly, the value of $N_{\mathrm{e}}$ for the energy-dependent DOS can be calculated using eqs. (8) and (1). We obtain the following analytical expression:

$$
N_{\mathrm{e}}=\left(\frac{T}{T_{\mathrm{e}}^{\prime}}\right)^{2} R^{4}\left[\left(\eta_{1}+\frac{1}{2}+\frac{2 \beta}{\pi}+\frac{3 \beta^{2}}{4}\right)+\left(2+4 \eta_{1}+\frac{8 \beta}{\pi}\right) \frac{\varepsilon}{R}\right]
$$

where the subscript $\mathrm{e}$ indicates electron-electron interactions. In these expressions for $N$, we define $T_{\mathrm{c}}^{\prime}=12 \alpha^{2} / \pi D_{0} k_{\mathrm{B}}$ and $T_{\mathrm{e}}^{\prime}=\left(48 \alpha^{2} / \pi D_{1} k_{\mathrm{B}}^{2}\right)^{1 / 2}$. Furthermore, $\eta_{1}$ is given as 


$$
\eta_{1}= \begin{cases}\frac{2}{3 \pi}, & \beta=1 \\ \frac{\beta}{\pi\left(\beta^{2}-1\right)}+\frac{2}{\pi\left(1-\beta^{2}\right)^{3 / 2}} \tan ^{-1}\left[\left(\frac{1-\beta}{1+\beta}\right)^{1 / 2}\right] & \beta<1 \\ \frac{\beta}{\pi\left(\beta^{2}-1\right)}-\frac{2}{\pi\left(\beta^{2}-1\right)^{3 / 2}} \tanh ^{-1}\left[\left(\frac{\beta-1}{\beta+1}\right)^{1 / 2}\right], & \beta>1\end{cases}
$$

In order to acquire eqns. (9) and (10), we approximated the derivative of the Fermi distribution with the Dirac delta function, and we let $f(\varepsilon-R \beta \cos \theta) \approx 1$ for $\varepsilon-R \beta \cos \theta<0$ and $f(\varepsilon-R \beta \cos \theta) \approx 0$ for $\varepsilon-R \beta \cos \theta>0$. Using eqns. (7), (9) and (10), we obtained the expressions for $P_{\mathrm{nn}}(R)$ for the constant DOS and energydependent DOS as

$$
\begin{aligned}
& P_{\mathrm{nn}}^{\mathrm{e}}(R)=\left(C_{1} R+C_{2} R^{2}+C_{3} R^{4}\right) \exp \left(-\frac{C_{2}}{3} R^{3}\right) \\
& P_{\mathrm{nn}}^{\mathrm{e}}(R)=\left(B_{1} R^{2}+B_{2} R^{3}+B_{3} R^{6}\right) \exp \left(-\frac{B_{2}}{4} R^{4}\right)
\end{aligned}
$$

where $C_{i}$ is defined as

$$
\begin{aligned}
& C_{1}=3\left(1+2 \eta_{1}\right) \frac{T}{T_{c}^{\prime}} \varepsilon, \\
& C_{2}=3\left(\frac{1}{2}+\frac{2 \beta}{\pi}+\eta_{1}\right) \frac{T}{T_{\mathrm{c}}^{\prime}}, \\
& C_{3}=-\frac{C_{1} C_{2}}{2},
\end{aligned}
$$

and $B_{i}$ as

$$
\begin{aligned}
& B_{1}=3\left(2 \pi+8 \beta+4 \xi_{1}\right)\left(\frac{T}{T_{\mathrm{e}}^{\prime}}\right)^{2} \varepsilon \\
& B_{2}=4\left(\frac{1}{2}+\frac{2 \beta}{\pi}+\eta_{1}+\frac{3 \beta^{2}}{4}\right)\left(\frac{T}{T_{\mathrm{e}}^{\prime}}\right)^{2}, \\
& B_{3}=-\frac{B_{1} B_{2}}{3}
\end{aligned}
$$

The expression for $R_{\mathrm{nn}}=R_{\mathrm{c}}$ is obtained from eqns. (6) and (12) for the constant DOS as

$$
R_{\mathrm{c}}=R_{\mathrm{c}}^{0}-R_{\mathrm{c}}^{1} \varepsilon, R_{\mathrm{c}}^{1}=\frac{\frac{1}{2}+\eta_{1}}{2 \beta / \pi+\frac{1}{2}+\eta_{1}},
$$

to first order in $\varepsilon$, where $\varepsilon$ is assumed to be very small, that is close to the Fermi level. Similarly, the expression $R_{\mathrm{nn}}=R_{\mathrm{e}}$ for the energy-dependent DOS is obtained as

$$
R_{\mathrm{e}}=R_{\mathrm{e}}^{0}-R_{\mathrm{e}}^{1} \varepsilon, R_{\mathrm{e}}^{1}=\frac{\frac{1}{2}+\eta_{1}+2 \beta / \pi}{\frac{1}{2}+\eta_{1}+2 \beta / \pi+3 \beta^{2} / 4},
$$

where $R_{\mathrm{c}}^{0}$ and $R_{\mathrm{e}}^{0}$ are written as 


$$
\begin{aligned}
& R_{\mathrm{c}}^{0}=\left(\frac{T}{T_{\mathrm{c}}}\right)^{-1 / 3}\left(\frac{1}{2}+\eta_{1}+\frac{2 \beta}{\pi}\right)^{-1 / 3} \\
& R_{\mathrm{e}}^{0}=\left(\frac{T}{T_{\mathrm{e}}}\right)^{-1 / 2}\left(\frac{1}{2}+\eta_{1}+\frac{2 \beta}{\pi}+\frac{3 \beta^{2}}{4}\right)^{-1 / 4} .
\end{aligned}
$$

We have now defined $T_{\mathrm{c}}=\left[\Gamma\left(\frac{4}{3}\right)\right]^{3} T_{\mathrm{c}}^{\prime}$ and $T_{\mathrm{e}}=\left[\Gamma\left(\frac{5}{4}\right)\right]^{2} T_{\mathrm{e}}^{\prime}$. Note that our expression for $R_{\mathrm{nn}}$ depends on the initial energy of the electrons. In our previous paper (Singh $e t$ al. 1996), we calculated $R_{\mathrm{nn}}$ by just assuming that $N=1$.

Expressions for $x_{\mathrm{f}}$ can be calculated for the constant DOS and energy-dependent DOS using eqns. (5) and (15)-(17) to give

$$
\begin{aligned}
& x_{\mathrm{f}}^{\mathrm{c}}=R_{\mathrm{c}}^{0} \frac{\eta_{3} / 3-\beta / 6-1 / 3 \pi}{\eta_{2} / 2+1 / 4+\beta / 2 \pi} \equiv R_{\mathrm{c}}^{0} G_{\mathrm{c}}(\beta), \\
& x_{\mathrm{f}}^{\mathrm{e}}=R_{\mathrm{e}}^{0} \frac{\eta_{3} / 2-1 / 2 \pi-\beta / 4-\beta^{2} / \pi}{\eta_{2}+1 / 2+\beta / \pi+\beta^{2} / 4} \equiv R_{\mathrm{e}}^{0} G_{\mathrm{e}}(\beta),
\end{aligned}
$$

where $\eta_{2}$ is defined as

$$
\eta_{2}= \begin{cases}\frac{1}{\pi}, & \beta=1 \\ \frac{2}{\pi\left(1-\beta^{2}\right)^{1 / 2}} \tan ^{-1}\left[\left(\frac{1-\beta}{1+\beta}\right)^{1 / 2}\right], & \beta<1 \\ \frac{2}{\pi\left(\beta^{2}-1\right)^{1 / 2}} \tanh ^{-1}\left[\left(\frac{\beta-1}{\beta+1}\right)^{1 / 2}\right], & \beta>1\end{cases}
$$

and $\eta_{3}$ is defined as

$$
\eta_{3}= \begin{cases}\frac{1}{3 \pi}, & \beta=1, \\ \frac{1}{\pi\left(1-\beta^{2}\right)}-\frac{2 \beta}{\pi\left(1-\beta^{2}\right)^{3 / 2}} \tan ^{-1}\left[\left(\frac{1-\beta}{1+\beta}\right)^{1 / 2}\right], & \beta<1, \\ \frac{1}{\pi\left(1-\beta^{2}\right)}+\frac{2 \beta}{\pi\left(\beta^{2}-1\right)^{3 / 2}} \tanh ^{-1}\left[\left(\frac{\beta-1}{\beta+1}\right)^{1 / 2}\right], & \beta>1\end{cases}
$$

Here, $x_{\mathrm{f}}^{\mathrm{c}}$ and $x_{\mathrm{f}}^{\mathrm{e}}$ have been evaluated at the Fermi level, that is at $\varepsilon=0$. Note that, at zero electric field, $x_{\mathrm{f}}^{\mathrm{c}}$ and $x_{\mathrm{f}}^{\mathrm{e}}$ become zero. From eqns. (4) and (18), the mobilities will be

$$
\begin{aligned}
& \mu_{\mathrm{c}}=\frac{\nu_{\mathrm{p}}}{2 \alpha E} R_{\mathrm{c}}^{0} G_{\mathrm{c}}(\beta) \exp \left(-R_{\mathrm{c}}^{0}+R_{\mathrm{c}}^{1} \varepsilon\right), \\
& \mu_{\mathrm{e}}=\frac{\nu_{\mathrm{p}}}{2 \alpha E} R_{\mathrm{e}}^{0} G_{\mathrm{e}}(\beta) \exp \left(-R_{\mathrm{e}}^{0}+R_{\mathrm{e}}^{1} \varepsilon\right)
\end{aligned}
$$

Finally, the conductivity of carriers can be obtained from the mobility as (Singh et al. 1994a,b)

$$
\sigma(T, E)=q k_{\mathrm{B}} T \int_{-\infty}^{\infty} D(\omega) f(\omega) \mu(\omega, T, E) \mathrm{d} \omega
$$


Using eqns. (21) and (22), we obtain the following analytical expressions of conductivity for the constant DOS and energy-dependent DOS, respectively:

$$
\begin{gathered}
\sigma_{\mathrm{c}}=\frac{c_{0} T G_{\mathrm{c}}(\beta)}{E}\left(\frac{R_{\mathrm{c}}^{0}+1}{R_{\mathrm{c}}^{1}}\right) \exp \left[-\left(\frac{T}{T_{\mathrm{c}}}\right)^{-1 / 3}\left(\frac{1}{2}+\frac{2 \beta}{\pi}+\eta_{1}\right)^{-1 / 3}\right], \\
\sigma_{\mathrm{e}}=\frac{c_{1} T^{2} G_{\mathrm{e}}(\beta)}{E}\left(\frac{R_{\mathrm{e}}^{0}+2}{\left(R_{\mathrm{e}}^{1}\right)^{2}}\right) \exp \left[-\left(\frac{T}{T_{\mathrm{e}}}\right)^{-1 / 2}\left(\frac{1}{2}+\frac{2 \beta}{\pi}+\eta_{1}+\frac{3 \beta^{2}}{4}\right)^{-1 / 4}\right],
\end{gathered}
$$

where $c_{0}$ and $c_{1}$ are given by

$$
\begin{aligned}
& c_{0}=\frac{q k_{\mathrm{B}} D_{0} \nu_{\mathrm{p}}}{4 \alpha}, \\
& c_{1}=\frac{q\left(k_{\mathrm{B}}\right)^{2} D_{1} \nu_{\mathrm{p}}}{4 \alpha} .
\end{aligned}
$$

The quantities $c_{0}$ and $c_{1}$ will be treated as parameters in this paper. Note that, in the present approach, the mobility is calculated as a function of energy with respect to the Fermi energy. Therefore it is straightforward to include extended states in the calculation of the conductivity. Note that the above expressions give the correct $T$ dependence (Mott and Davis 1971, Shklovskii 1973, Mott 1974, Efros and Shklovskii 1985). For $\beta \gg 1$, eqn. (23) reduces to $\ln \left(\sigma_{\mathrm{c}} / \sigma_{\mathrm{c}}^{0}\right) \propto \beta^{-1 / 3}$ and equation (24) becomes $\ln \left(\sigma_{\mathrm{e}} / \sigma_{\mathrm{e}}^{0}\right) \propto \beta^{-1 / 2}$. For the constant DOS we get agreement with the theory of Shklovskii (1976) for strong fields. Apsley and Hughes (1975) and Pollak and Riess (1976) do not explicitly calculate the two-dimensional field dependence.

\section{§ 3. RESULTS AND DISCUSSION}

Kabasawa et al. (1992) measured a current-voltage conductivity for a $2.0 \mu \mathrm{m}$ PBCO-based S-N-S junction at $T=4.2 \mathrm{~K}$. We want to compare our theory with the above experiment. In fitting the $\mathrm{I}-\mathrm{V}$ data, we used $\sigma \equiv J / E$ (Liu and Soonpaa 1993). In order to use either eqn. (23) or eqn. (24) to fit such data, we need values for $T_{\mathrm{c}}$ and $T_{\mathrm{e}}$. These parameters are taken from the work of Singh et al. $(1994 \mathrm{a}, \mathrm{b})$ for a PBCO thin film. That article gives values of $T_{\mathrm{c}}^{\prime}=2.7 \times 10^{4} \mathrm{~K}$ and $T_{\mathrm{e}}^{\prime}=1.3 \times 10^{3} \mathrm{~K}$, from which one can get $T_{\mathrm{c}}=1.9 \times 10^{4} \mathrm{~K}$ and $T_{\mathrm{e}}=1.1 \times 10^{3} \mathrm{~K}$. The thin-film data in the aforementioned paper shows a phase transition from constant-DOS conductivity to electron-electron conductivity at about $10.5 \mathrm{~K}$. The $\mathrm{I}-\mathrm{V}$ experiment is performed at $4.2 \mathrm{~K}$ where electron-electron interactions are important. Therefore we used eqn. (24) to fit this experiment. The theoretical curve along with experimental points are presented in fig. 1 . Using the value of $T_{\mathrm{e}}$ given above, we were able to fit the data well be choosing $1 / \alpha=8 \mathrm{~nm}$. This is similar to the value of $1 / \alpha$ found in previous work (Kabasawa et al. 1993, Singh et al. 1994a,b) and gives a value of $D_{1}=7.4 \times 10^{56} \mathrm{~J}^{-2} \mathrm{~m}^{-2}$. When using the constant DOS expression (eqn. (23)), we were not able to fit the data as well. The Kabasawa et al. (1993) I-V data was also more in agreement with eqn. (24) than with eqn. (23) (not depicted). Although we had fitted these data previously using a constant DOS (Singh et al. 1996), that was done using an expression based on Mott's conductivity devoid of all pre-exponential field dependence. It would seem these pre-exponential factors can have a noticeable effect. 
Fig. 1

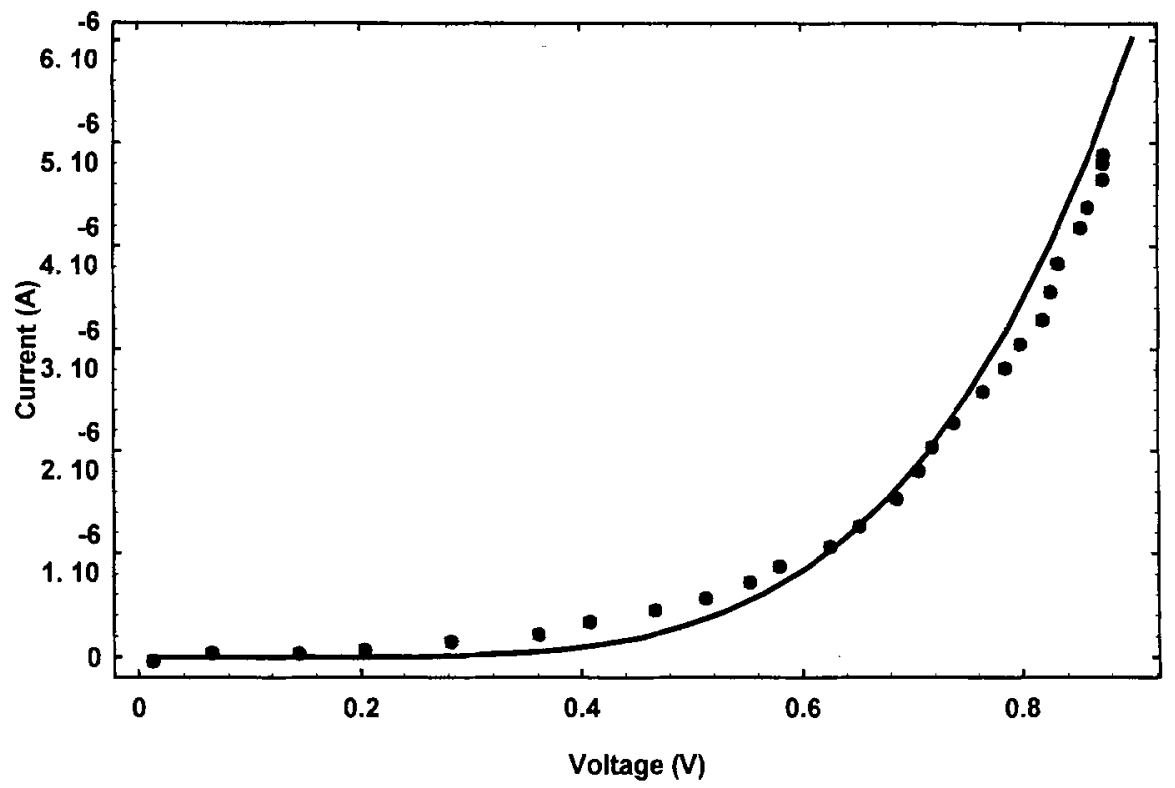

Current-voltage data of a PBCO-based S-N-S junction. The solid curve represents eqn. (24) with $1 / \alpha=8 \mathrm{~nm}$ and $T_{\mathrm{e}}=1.1 \times 10^{3} \mathrm{~K}$.

Since our theory is generally valid for any two-dimensional VRH system, we used the electric-field and temperature-dependent data of Liu and Soonpaa (1993) for $\mathrm{Bi}_{14} \mathrm{Te}_{11} \mathrm{~S}_{10}$. This allows an independent determination of the parameters $D_{0}, D_{1}$ and $\alpha$ for their compound, which are not known. To fit the temperature-dependent data, we used the conductivity expressions (23) and (24) for constant-DOS and electron-electron interactions respectively. The results are shown in fig. 2 , where we observe again a phase transition between the electron-electron interaction regime and the constant-DOS regime. The cross-over temperature is about $6.5 \mathrm{~K}$. We got values of $T_{\mathrm{c}}=1.8 \times 10^{3} \mathrm{~K}$ and $T_{\mathrm{e}}=7.4 \times 10^{2} \mathrm{~K}$. We found we could fit the $J$ against $E$ data better using eqn. (24) rather than eqn. (23). The results are shown in fig. 3. This is not surprising since the experiment was conducted at $2.38 \mathrm{~K}$ where electron-electron interactions would become important. From this fitting, we found that $1 / \alpha=60 \mathrm{~nm}$, and thus $D_{1}=2.7 \times 10^{55} \mathrm{~J}^{-2} \mathrm{~m}^{-2}$. The value of $\alpha$ is not far from the values found by Liu and Soonpaa, which were in the neighbourhood of $70 \mathrm{~nm}$. To fit all the experiments, we treated $c_{0}$ and $c_{1}$ as parameters. We found that they varied between samples and between research groups.

\section{ACKNOWLEDGMENTS}

The authors would like to thank H. H. Soonpaa and G. van Ancum for sending complete data and explanations for the separate experiments that they conducted. One of us (M.S.) is grateful to the Natural Sciences and Engineering Research Council of Canada for financial support in the form of a research grant. 
Fig. 2

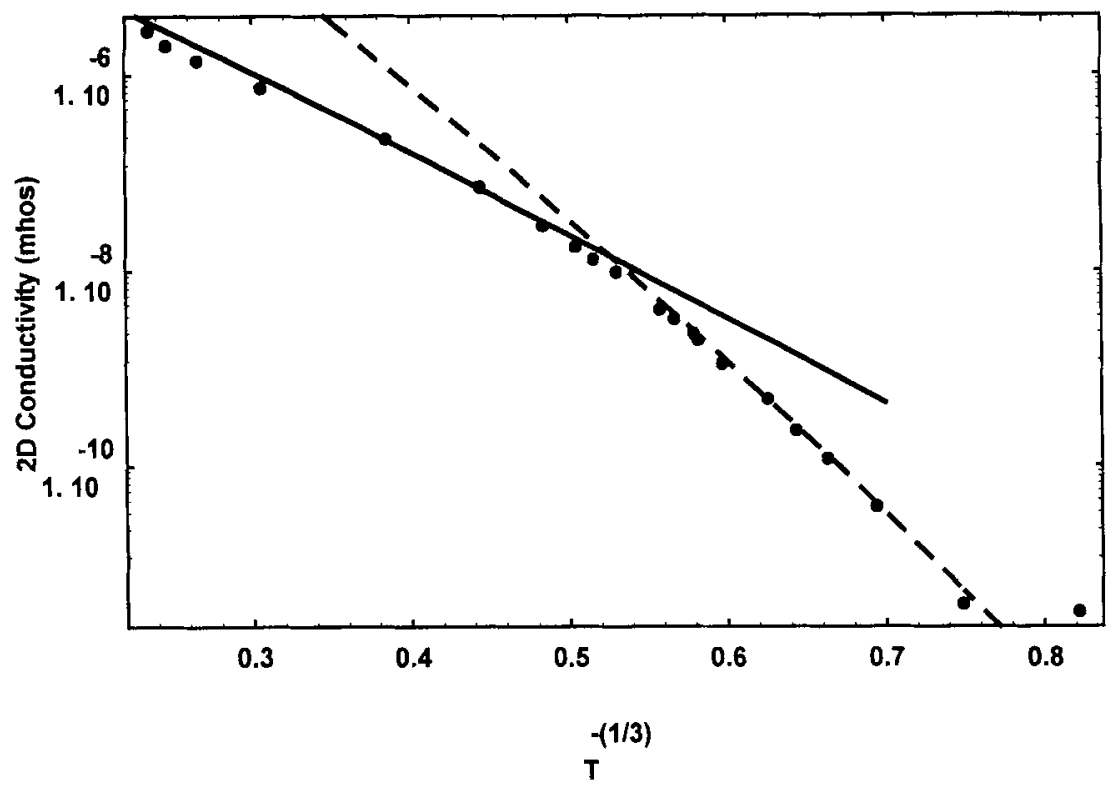

Plot of conductivity against temperature for a $\mathrm{Bi}_{14} \mathrm{Te}_{11} \mathrm{~S}_{10}$ thin film. The full circles represent experimental results. The solid and broken curves correspond to constant-DOS and electron-electron interactions respectively with $T_{\mathrm{c}}=1.8 \times 10^{3} \mathrm{~K}$ and $T_{\mathrm{e}}=$ $7.4 \times 10^{2} \mathrm{~K}$.

Fig. 3

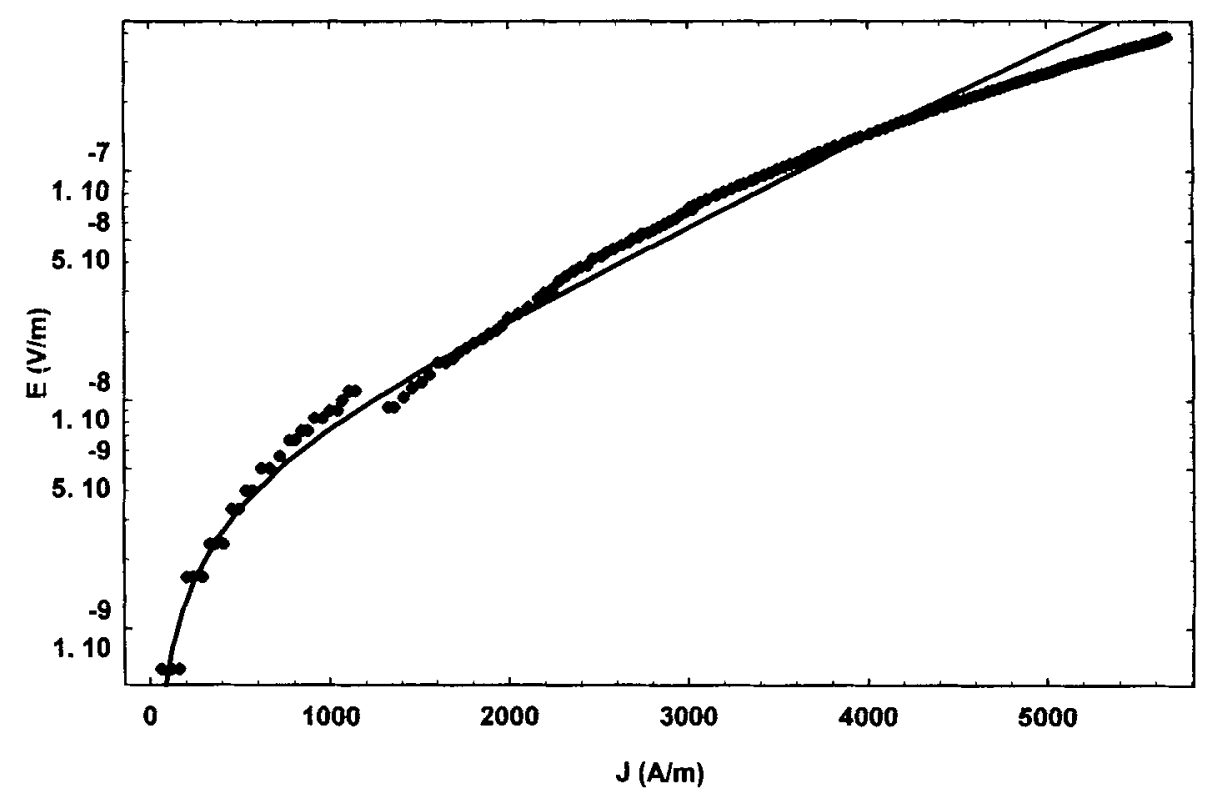

Current density against electric field data. The solid curve represents eqn. (24) with $1 / \alpha=60 \mathrm{~nm}$ and $T_{\mathrm{e}}=7.4 \times 10^{2} \mathrm{~K}$. 


\section{REFERENCES}

Anderson, P.W., 1988, Frontiers and Borderlines in many Particle Physics, Verenna Lecture, edited by J.R. Schrieffer and R.A. Broglia (Amsterdam: North-Holland).

Apsley, N., and Hughes, H.P., 1974, Phil. Mag., 30, 963; 1975, Ibid., 31, 1327.

Bociuslavskij, Yu., M., Verhoeven, M.A.J., Roesthuis, F.J.G., Gerritsma, G.J., and Rogalla, H., 1994, Physica B, 194, 1115.

Efros, A.L., and ShKLovskir, B.I., 1985, Electron-Electron Interaction in Disordered Systems (Amsterdam: North-Holland).

Forro, L., 1995, Int. J. mod. Phys. B, 8, 829.

Hu, X.L., Van Keuls, F. W., Carmi, Y., Jiang, H.W., and Dahm, A.J., 1995, Solid St. Commun., 96, 65.

Kabasawa, U., Tarutani, Y., Tsukamoto, A., Fukazawa, T., Hiratani, M., and Takagi, K., 1992, Physica C, 194, 261.

Kabasawa, U., Tarutant, Y., Okamoto, M., Fukazawa, T., Tsukamoto, A., Hiratani, M., and TAKAGI, K., 1993, Phys. Rev. Lett., 70, 1700, and references therein.

Koyanagi, M., Kashiwaya, S., Matsuda, M., Short, A., and Kajimura, K., 1994, Physica B, $194,2155$.

Liv, G., and SoonpaA, H.H., 1993, Phys. Rev. B, 48, 5682.

Mason, W., Kravchenko, S.V., Bowker, G.E., and Furneaux, J.E., 1995, Phys. Rev. B, 52, 7857.

MotT, N.F., and Davis, E.A., 1971, Electron Processes in Non-crystalline Materials (London: Oxford University Press).

MotT, N.F., 1974, Metal-Insulator Transition (London: Taylor \& Francis)

Pollak, M., and Riess, I., 1976, J. Phys. C, 9, 2339.

SeONG, D., Lee, S., and Youm, D., 1990, Solid St. Commun., 76, 1341.

ShkLovskiI, B.I., 1973, Soviet Phys. Semicond., 6, 1964; 1976, Ibid., 10, 855; 1991, Hopping Transport in Solids, edited by M. Pollak and B.I. Shklovskii (Amsterdam: NorthHolland) and references therein.

Singh, M., and Sinha, K.P., 1989, Solid St. Commun., 70, 149.

Singh, M., Tarutani, Y., Kabasawa, U., and Takagi, K., 1994a, Phys. Rev. B, 50, 7007; 1994b, Solid St. Commun., 89, 255, and references therein.

Singh, M., Thompson, R.B., and Dumas, O., 1996, Phys. Rev. B, 53, 6806.

Sinha, K.P., and Singh, M., 1988, J. Phys. C, 21, L231.

van Ancum, G.K., Verhoeven, M.A.J., Bl.ANK, D.H.A., and Rogalla, H., 1995, Phys. Rev. B, 52, 5598. 